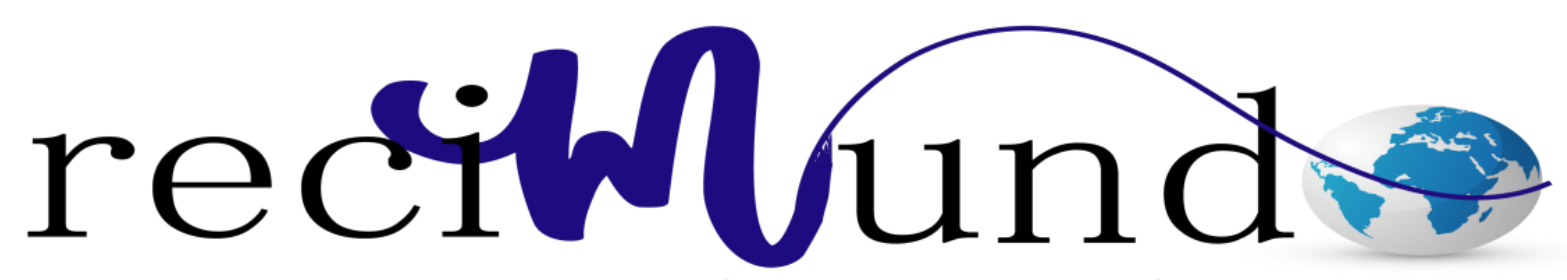

Revista Cientifica Mundo de la Investigación y el Conocimiento

Ana María Viteri Rojas a; Katherine Angélica Sánchez Alcantara b; Andrea Alexandra Saltos Román ${ }^{\mathrm{c}}$

Complicaciones frecuentes inmediatas en paciente de 20-29 años sometidos a manga gástrica laparoscópica del Hospital Teodoro Maldonado

Immediate frequent complications in a 20-29 year old patient undergoing laparoscopic gastric sleeve at Teodoro Maldonado Hospital

Revista Científica Mundo de la Investigación y el Conocimiento. Vol. 3 núm.3, septiembre, ISSN: 2588-073X, 2019, pp. 887-918

DOI: $10.26820 /$ recimundo/3.(3).septiembre.2019.887-918

URL: $\underline{\text { http://recimundo.com/index.php/es/article/view/554 }}$

Código UNESCO: 3205 Medicina Interna

Tipo de Investigación: Artículo de Revisión

Editorial Saberes del Conocimiento

Recibido: 15/05/2019

Aceptado: 23/06/2019

Publicado: 30/09/2019

Correspondencia: anyviten031@hotmail.com

a. Especialista en Cirugía General; Doctora en Medicina y Cirugía; Investigador Independiente; Guayaquil, Ecuador; anyviten031@ hotmail.com

b. Médico; Investigador Independiente; Guayaquil, Ecuador;sanchezalcantara911@ @otmail.com

c. Médico, Residente del Hospital Gineco-Obtetrico Universitario, Investigador Independiente; Guayaquil, Ecuador; andrea.saltos@ hotmail.com 


\section{Complicaciones frecuentes inmediatas en paciente de 20-29 años sometidos a manga gástrica laparoscópica del Hospital Teodoro Maldonado}

Vol. 3, núm. 3., (2019)

Ana María Viteri Rojas; Katherine Angélica Sánchez Alcantara; Andrea Alexandra Saltos Román

\section{RESUMEN}

La sociedad en los últimos años ha estado sumergida en un proceso evolutivo que ha ayudado a minimizar las necesidades de sus ciudadanos a través de herramientas y técnicas más fáciles de manejar y producir. Esto trae como consecuencia que los individuos aumenten sus horas de trabajo permitiendo que se forme el sedentarismo y una alimentación basada en altas calorías y azucares. Esto produce la obesidad y a su vez enfermedades colaterales que nacen de esta enfermedad. Existen diversos tratamientos, pero el más fundamental es la motivación que es función de alta autoestima. También está el cambio de dieta y la ejercitación física. Existen tratamientos quirúrgicos como la cirugía bariátrica. Entre estas operaciones bariátricas se encuentra la manga gástrica laparoscópica. Esta se encarga de seccionar el estómago a través de grapas que reducen su volumen y que generar menor ansiedad en la ingesta de alimentos. El objetivo general de esta investigación es analizar las complicaciones frecuentes inmediatas en pacientes de 20-29 años sometidos a manga gástrica laparoscópica del Hospital Teodoro Maldonado. La metodología empleada es a través de investigaciones de tipo documental y bibliográfica. Los resultados obtenidos se basaron en las características de la cirugía de manga gástrica laparoscópica, complicaciones postoperatorias de la misma y análisis de estas operaciones en el Hospital Teodoro Maldonado. Como conclusión arrojo que los adultos jóvenes, sufren de complicaciones postoperatoria principalmente de fistula gástrica y reflujo gastroesofágico, donde la primera es por consecuencia de pérdida de líquido intestinal la cual de

difunde y se acumula formando una fistula en la piel que puede tender a peritonitis y la segunda es por la falla del mecanismo que regula el antirreflujo gástrico del esófago. Esta dificultad postoperatoria se incrementa si el paciente joven sufre de hipertensión arterial más si es consumidor de alcohol y cigarrillos.

Palabras Claves: Alimentación; Sedentarismo; Obesidad Cirugía Bariátrica; Manga Gástrica Laparoscópica. 


\title{
Complicaciones frecuentes inmediatas en paciente de 20-29 años sometidos a manga gástrica laparoscópica del Hospital Teodoro Maldonado
}

Vol. 3, núm. 3., (2019)

Ana María Viteri Rojas; Katherine Angélica Sánchez Alcantara; Andrea Alexandra Saltos

Román

\begin{abstract}
Society in recent years has been immersed in an evolutionary process that has helped minimize the needs of its citizens through tools and techniques that are easier to handle and produce. This results in individuals increasing their work hours by allowing a sedentary lifestyle and a diet based on high calories and sugars. This produces obesity and in turn collateral diseases that arise from this disease. There are various treatments, but the most fundamental is the motivation that is a function of high self-esteem. There is also the change of diet and physical exercise. There are surgical treatments such as bariatric surgery. Among these bariatric operations is the laparoscopic gastric sleeve. This is responsible for sectioning the stomach through staples that reduce its volume and generate less anxiety in food intake. The general objective of this research is to analyze the immediate frequent complications in patients aged 20-29 years undergoing laparoscopic gastric sleeve of Teodoro Maldonado Hospital. The methodology used is through documentary and bibliographic research. The results obtained were based on the characteristics of laparoscopic gastric sleeve surgery, postoperative complications and analysis of these operations at the Teodoro Maldonado Hospital. En conclusion, it shows that young adults suffer from postoperative complications mainly of gastric fistula and gastroesophageal reflux, where the first is due to loss of intestinal fluid which diffuses and accumulates forming a fistula in the skin that can tend to peritonitis and the second is due to the failure of the mechanism that regulates the gastric antireflux of the esophagus. This postoperative difficulty is increased if the young patient suffers from high blood pressure more if he is a consumer of alcohol and cigarettes.
\end{abstract}

Key Words: Food; Sedentary Lifestyle; Obesity; Bariatric Surgery; Laparoscopic Gastric Sleeve. 


\section{Complicaciones frecuentes inmediatas en paciente de 20-29 años sometidos a manga gástrica laparoscópica del Hospital Teodoro Maldonado}

Vol. 3, núm. 3., (2019)

Ana María Viteri Rojas; Katherine Angélica Sánchez Alcantara; Andrea Alexandra Saltos Román

\section{Introducción.}

El desarrollo de las sociedades va enmarcado en un sinfín de objetivos que han buscado satisfacer las necesidades hasta sobrepasar las expectativas del individuo. Sin duda, la globalización ha incrementado el desarrollo en todas las aristas. Una de ellas es el consumo excesivo de alimentos no favorables a la salud de las personas. Esto produce un efecto negativo debido al incremento del peso corporal produciendo así la obesidad. Esta enfermedad es considerada una de los más grandes males de nuestros tiempos. De acuerdo a la Organización Mundial de la Salud, la obesidad es una enfermedad crónica, caracterizada por el aumento de la grasa corporal, asociada a mayor riesgo para la salud(Moreno, 2012).

"Es una enfermedad de etiología multifactorial de curso crónico en la cual se involucran aspectos genéticos, ambientales y de estilo de vida que conducen a un trastorno metabólico. Se caracteriza por un balance positivo de energía, que ocurre cuando la ingestión de calorías excede al gasto energético ocasionando un aumento en los depósitos de la grasa corporal y por lo tanto ganancia de peso”. (García, 2004, pág. 88)

El incremento en el consumo de calorías hace que aumente la masa corporal, produciendo un cambio físico en el individuo, pero a su vez ocasiona que la salud disminuya drásticamente. La obesidad se define por un índice de masa corporal (IMC) depeso $(\mathrm{kg}) /$ talla $\left(\mathrm{m}^{2}\right) \geq 30$, así como también unacircunferencia de la cintura mayor de102 centímetros en hombres, y de 88centímetros en mujeres esindicativa de obesidad(Bersh, 2006). 


\section{Complicaciones frecuentes inmediatas en paciente de 20-29 años sometidos a manga gástrica laparoscópica del Hospital Teodoro Maldonado}

Vol. 3, núm. 3., (2019)

Ana María Viteri Rojas; Katherine Angélica Sánchez Alcantara; Andrea Alexandra Saltos

Román

"La obesidad resulta cuando se aumenta el tamaño y el número de las células grasas (adipocitos) de una persona. Un individuo adulto de tamaño y peso promedio tiene entre 25 y 30 billones de adipocitos, pero cuando su peso se eleva, estas células aumentan de tamaño inicialmente y luego su númerose multiplica”.(Daza, 2002, pág. 73)

Los adipocitos juegan un papel importante en el incremento de la masa corporal debido a que un aumento de su volumen hace que se manifieste en el aumento de la masa corporal. El peso de esta célula grasa es muy poco, pero al considerarse billones esta situación cambia y por ende aumenta el peso del individuo. Aunque no es práctico contar ymedir los adipocitos de una persona, laciencia ha logrado diseñar métodos fácilespara determinar la obesidad(Daza, 2002).

Este incremento de la obesidad es imputado al estilo de vida de la persona. La ingesta de calorías a través de los alimentos, así como un excesivo sedentarismo y la comodidad que ha desarrollado el mundo globalizado a servido de catalizador para esta enfermedad. En la Tabla 1, se puede observar las características que predominan en el desarrollo de la obesidad en función de la alimentación, actividad física y aspectos socioculturales.

\section{Tabla 1.}

Características que predominan en el desarrollo de la obesidad en función de la alimentación, actividad física y aspectos socioculturales

\begin{tabular}{cl}
\hline Factores & \multicolumn{1}{c}{ Características } \\
\hline \multirow{3}{*}{ Alimentación } & $\begin{array}{l}\text { Mayor consumo de alimentos de alta densidad energética y bajo } \\
\text { contenido de nutrientes y fibra. }\end{array}$ \\
\cline { 2 - 2 } & $\begin{array}{l}\text { Alto consumo de alimentos y bebidas con azúcares simples e } \\
\text { hidratos de carbono refinados. }\end{array}$ \\
\cline { 2 - 2 } & $\begin{array}{l}\text { Alto consumo de grasas saturadas, ácidos grasos } \\
\text { monoinsaturados "trans" y colesterol. }\end{array}$ \\
\cline { 2 - 2 } & Aumento del tamaño de las raciones de alimentos, especialmente \\
\hline
\end{tabular}




\section{Complicaciones frecuentes inmediatas en paciente de 20-29 años sometidos a manga gástrica laparoscópica del Hospital Teodoro Maldonado}

Vol. 3, núm. 3., (2019)

Ana María Viteri Rojas; Katherine Angélica Sánchez Alcantara; Andrea Alexandra Saltos Román

\begin{tabular}{|c|c|}
\hline & en restaurantes y "cadenas de alimentos rápidos". \\
\hline & Menor consumo de vegetales y frutas frescas. \\
\hline \multirow{6}{*}{ Actividad física } & Trabajo con menor esfuerzo físico. \\
\hline & Uso cada vez mayor de transporte automotor. \\
\hline & $\begin{array}{l}\text { Automatización de los vehículos y reducción del gasto energético } \\
\text { en la operación de maquinarias y vehículos. }\end{array}$ \\
\hline & Uso de ascensores y escaleras eléctricas. \\
\hline & Reducción del tiempo dedicado a jugar al aire libre. \\
\hline & Preferencia por juegos electrónicos y televisión. \\
\hline \multirow{4}{*}{$\begin{array}{c}\text { Aspectos } \\
\text { socioculturales }\end{array}$} & $\begin{array}{l}\text { Aceptación de la obesidad como expresión de salud y belleza y } \\
\text { no de enfermedad. }\end{array}$ \\
\hline & Considerar a la obesidad como sinónimo de afluencia económica. \\
\hline & Falta de concientización de los riesgos de sobrepeso y obesidad. \\
\hline & Pobre nivel educativo en nutrición en todoslos niveles. \\
\hline
\end{tabular}

Fuente: (Barrientos-Pérez \& Flores-Huerta, 2008)

La alimentación siempre ha sido el factor más preponderante para el incremento de la masa corporal, y es el consumo de azúcar el medio que impulsa el aumento de las calorías. La OMS recomienda reducir el consumo de azúcares libres a lo largo del ciclo de vida ya que la idea no es criminalizar al azúcar debido a que ésta ofrece también muchos otros beneficios(San Lucas, Montanero, Panchana, Robles, \& Vayas, 2018).

El aspecto sociocultural es importante ya que depende de la sociedad donde habita la persona que sufre esta enfermedad. Cada cultura es diferente y aborda este tema deotras perspectivas. Las familias juegan un papel determinante ante esta problemática. Son determinantes para generar una condición de obesidad en los niños, está ampliamente documentada la relación directa que hay entre padres obesos y la probabilidad de que los hijos también presenten obesidad o sobre peso(Ochoa, y otros, 2018). Dentro de este factor sociocultural estas las políticas sociales que están alrededor de los individuos, tal cual se observa en la Figura 1. 


\section{Complicaciones frecuentes inmediatas en paciente de 20-29 años sometidos a manga gástrica laparoscópica del Hospital Teodoro Maldonado}

Vol. 3, núm. 3., (2019)

Ana María Viteri Rojas; Katherine Angélica Sánchez Alcantara; Andrea Alexandra Saltos

Román

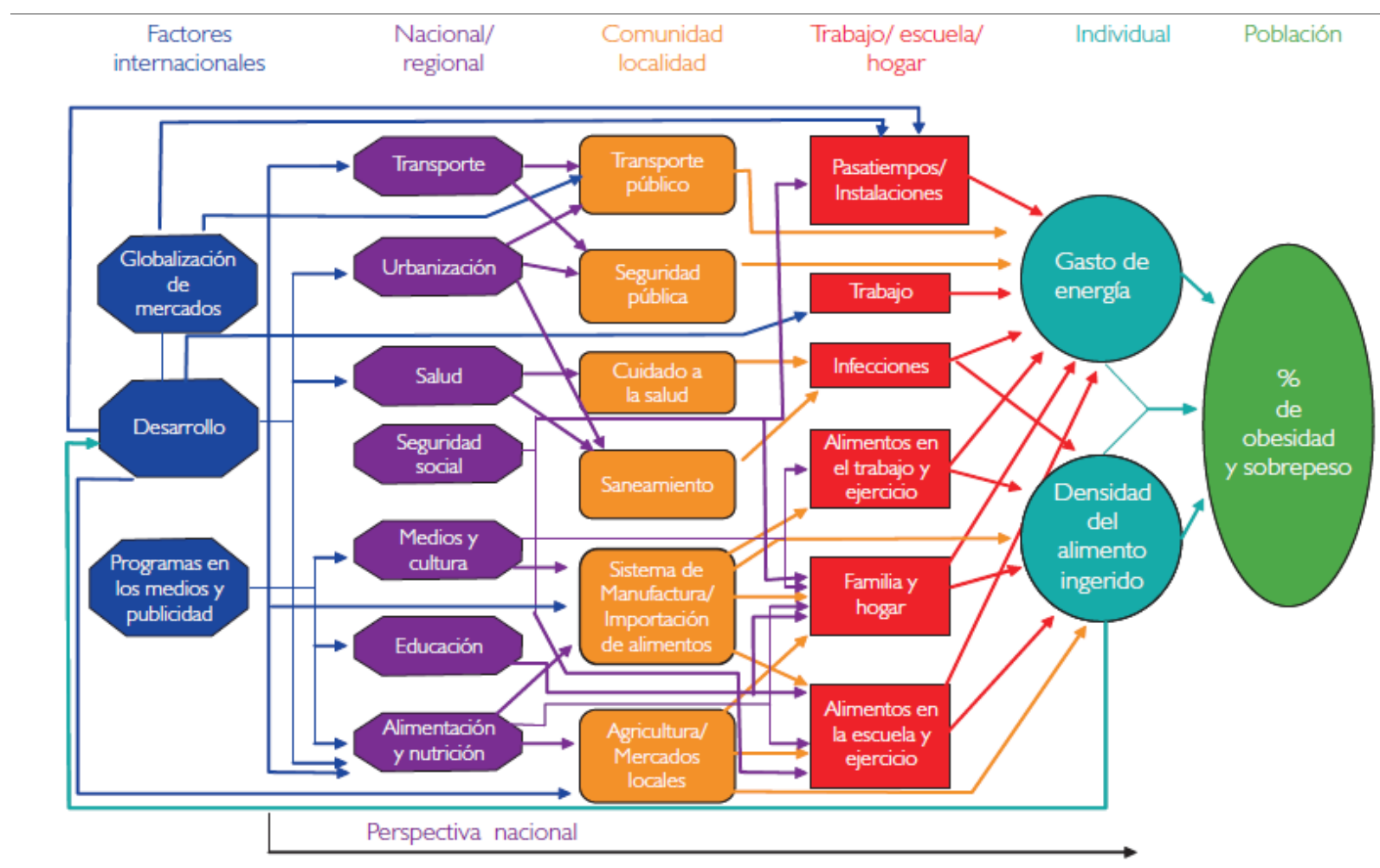

Figura 1. Políticas sociales y procesos que influencian la prevalencia de la obesidad en la población.

Fuente: (Barrientos-Pérez \& Flores-Huerta, 2008)

No solo es la salud física, también esta enfermedad puede producir en los individuos problemas psicológicos que afectan su calidad de vida.

"Es más común encontrar alteraciones de la personalidad y problemas psiquiátricos en personas obesas que en las de peso normal. Entre las alteraciones referidas se encuentran: agorafobia, depresión mayor, fobia simple, bulimia y dependencia del tabaco. Así mismo, se han apreciado desórdenes de personalidad relacionados con conductas excéntricas, dramáticas, una elevada ansiedad, conductas de evasión y agresión". (Velásquez \& Alvarenga, 2001, pág. 92) 


\section{Complicaciones frecuentes inmediatas en paciente de 20-29 años sometidos a manga gástrica laparoscópica del Hospital Teodoro Maldonado}

Vol. 3, núm. 3., (2019)

Ana María Viteri Rojas; Katherine Angélica Sánchez Alcantara; Andrea Alexandra Saltos Román

Esta enfermedad puede producir otros factores de riesgo de la enfermedad coronaria, tales como la hipertensión, la dislipidemia y la diabetes, aunque por mucho tiempo no fue bien definida como un factor de riesgo de la enfermedad coronaria debido a su marcada heterogeneidad (Scarsella \& Després, 2003).Las complicaciones colaterales por efecto de la obesidad se detallan en la Tabla 2.

\section{Tabla 2.}

Posibles signos clínicos en el paciente obeso y sus causas probables

\begin{tabular}{|c|c|}
\hline Signo clínico & Pensar en... \\
\hline Dolor abdominal recurrente y vago & Hígado graso no alcohólico \\
\hline Dolor abdominal epigástrico y regurgitación & Reflujo gastroesofágico \\
\hline Dolor y distensión abdominal & Constipación \\
\hline Dolor en el cuadrante superior derecho o epigástrico y vómitos & Colelitiasis \\
\hline Dolor en cadera, muslo y/o dificultad o dolor en la marcha & Necrosis de la cabeza femoral \\
\hline Dolor en rodilla y tobillo & Enfermedad de Blount \\
\hline Dolor de piernas y columna & Pies planos y escoliosis, respectivamente \\
\hline Cefalea, visión borrosa, vómitos & Pseudotumor cerebrii \\
\hline Irritación en pliegues & Micosis o sobreinfección bacteriana \\
\hline Respiración bucal & Apneas obstructivas \\
\hline Acantosis nigricans & Hiperinsulinismo/Insulinorresistencia \\
\hline $\begin{array}{l}\text { Tanner adelantado en mujeres y más lento en varones. } \\
\text { Seudoginecomastia en varones }\end{array}$ & Obesidad \\
\hline Hirsutismo, acné y/o trastornos menstruales & Síndrome del ovario poliquístico \\
\hline Xantelasma & Dislipidemia \\
\hline Palidez & Anemia \\
\hline Estrías rojizas & Síndrome de Cushing \\
\hline $\begin{array}{l}\text { Fascies peculiar, dismorfias, } \\
\text { problemas neurólogicos, hipogonadismo }\end{array}$ & Síndrome genético asociado a obesidad \\
\hline
\end{tabular}

Fuente: (Nutrición, 2011)

Sin duda alguna, el individuo debe contrarrestar esta enfermedad a través de diversos tratamientos. El tratamiento de la obesidad instaurada no es fácil y en muchas ocasiones conduce al fracaso, por un lado, porque es muy difícil erradicar los hábitos nutricionales y estilos de vida del niño y de la familia (Yeste, y otros, 2008). 


\section{Complicaciones frecuentes inmediatas en paciente de 20-29 años sometidos a manga gástrica laparoscópica del Hospital Teodoro Maldonado}

Vol. 3, núm. 3., (2019)

Ana María Viteri Rojas; Katherine Angélica Sánchez Alcantara; Andrea Alexandra Saltos

Román

Como la obesidad es difícil de controlar debido a factores que están alrededor de la persona entonces es necesario que exista un compromiso que impulse a desarrollar los tratamientos a realizar. La motivación es el principal factor para lograr un cambio conductual, debido a que se puede promover conductas saludables, favorece sentimientos positivos y mejora los resultados clínicos, pero si no, disminuye la autoestima e influye sobre futuros esfuerzos para mejorar(Nutrición, 2011).

El tratamiento en la parte de alimentación es muy importante porque ayuda a que los adipocitos no crezcan y por ende la masa corporal se mantenga en su peso ideal. Se debe aprender a ingerir los alimentos que ayuden a una mejor salud en el individuo. Comprar alimentos saludables; conocer los grupos de alimentos y sus características; planificar y organizar un menú semanal balanceado, completo y variado; consumir frutas y verduras como mínimo 5 porciones diarias; consumir fibra; y limitar bebidas azucaradas y alimentos hipercalóricos(Nutrición, 2011).

Otro de los mecanismos para mejorar y poder reducir de peso es la implementación de actividades físicas a diario. Los efectos de este tratamiento más una ingesta de alimentos balanceada produce buenos resultados. Los programas de actividad física deben tener una duración mínima de 12 semanas, donde se realice durante un tiempo de 30 min diarios. en adelante, a través de ejercicio aeróbico o entrenamiento con sobrecargas(Luque, García-Martos, Gutiérrez, \& Vallejo, 2010).

Todos estos tratamientos reflejan resultados positivos en poco tiempo pero que si no son tomados en cuenta por especialista en el área pueden traer contradicciones. Los médicos acoplan 


\section{Complicaciones frecuentes inmediatas en paciente de 20-29 años sometidos a manga gástrica laparoscópica del Hospital Teodoro Maldonado}

Vol. 3, núm. 3., (2019)

Ana María Viteri Rojas; Katherine Angélica Sánchez Alcantara; Andrea Alexandra Saltos Román

estos regímenes según sea el caso a través de un estudio completo de la salud del paciente. Es evidente que con los nuevos hallazgos y laevolución de la medicina han surgido nuevas técnicas y procedimientos que cada vez hacen máseficaz la forma de tratar el sobrepeso y combaten patologías que ponen enriesgo la salud del paciente(Viteri, y otros, 2019).

Existen tratamientos quirúrgicos cuando ya el sobrepeso es extremo, es decir cuando existe una obesidad mordica. Aunado a la alimentación y ejercitación se debe realizar cirugías que permitan un aumento en la calidad de vida del paciente. Una de ellas es la cirugía bariátrica. Esta proviene del antiguo griego cuyo término "bari" significa "gordo" y, por ende, este campo de la cirugía así llamada se enfoca en obtener una disminución de peso en forma sostenida y consecuente revertir la comorbilidad presente (Arriagada Solar, 2015).

"La cirugía bariátrica es una cirugía funcional o metabólica y no debe considerarse como una "cirugía estética". El paciente debe entender que, aunque los beneficios estéticos son importantes, no son el objetivo último de la cirugía. La meta no es alcanzar el peso ideal, que por otro lado sólo se consigue en algo más de la mitad de los casos. Tampoco debe considerarse como una "cirugía endocrina", pues no se actúa sobre glándulas de secreción interna. El propósito de esta cirugía es la disminución de la morbimortalidad que acompaña a la obesidad. Es decir, la pérdida de peso y, en consecuencia, la mejoría de los problemas de salud relacionados con la obesidad, serían los objetivos últimos de la intervención quirúrgica”.(Rodríguez, Mora, \& Palacios, 2013, pág. 2020)

Este tipo de tratamiento quirurgico porporciona cambios gastroinstestinales, anatomico y fincional, que buscan disminuir la ingesta de alimentos y la mala ansorción de los alimentos. La 


\section{Complicaciones frecuentes inmediatas en paciente de 20-29 años sometidos a manga gástrica laparoscópica del Hospital Teodoro Maldonado}

Vol. 3, núm. 3., (2019)

Ana María Viteri Rojas; Katherine Angélica Sánchez Alcantara; Andrea Alexandra Saltos

Román

reducción de la ingesta alimentaria y/o una malabsorciónde nutrientes pueden comprometer el estadonutricional del paciente, dando lugar a una malnutriciónproteicoenergética o al déficit selectivo dealgunos micronutrientes(Ocón Bretón, Pérez Naranjo, Gimeno Laborda, Benito Ruesca, \& García Hernández, 2005).El éxito se logra con una pérdida del exceso de peso superior al $50 \%$ a los 5 años, donde los primeros meses ocurre una rápida pérdida de peso estabilizándose a los 18-24 meses y con una posterior reganancia entre los 2-5 años(Solórzano, Flores, \& Ávila, 2019).

Esta cirugia bariatrica es especial si se trata de personas jovenes debido a sus multiples factores que integran la enfermedad de sobrepeso. La definición de la identidad, la estructura y exigencias del entorno educacional, la transición desde la dependencia parental hacia la autonomía, la influencia que tiene la instauración de hábitos, actitudes y estado de salud en la etapa adulta son algunos parámetros (Cruzat-Mandich, Díaz-Castrillón, García, \& Ulloa, 2018). Se debe tener estudios medícos y psicologicos de su comportamiento y de su forma de vida con el fin de poder lograr unos resultados positivos al final del tratamiento.

El futuro de estas cirugias es bastante prometedor. Se generan cada día muchos conocimientos alrededor de esta problemático y con ello infinida de soluciones a través de estas técnicas quirurgicas o tratamientos farmacologicos. Implantación de bandas, gastrectomías en manga, cirugías intraluminales y laparoscópicas, marcapasos gastrointestinal, o incluso a nivel cerebral, tendrán un importante rol, así como también el desarrollo de productos químicos o genéticamente modificados, tenderán a imitar los principios de la cirugía bariátrica(Fobi, 2010). 


\section{Complicaciones frecuentes inmediatas en paciente de 20-29 años sometidos a manga gástrica laparoscópica del Hospital Teodoro Maldonado}

Vol. 3, núm. 3., (2019)

Ana María Viteri Rojas; Katherine Angélica Sánchez Alcantara; Andrea Alexandra Saltos Román

De estas técnicas una de las más importante en estos ultimos años, y que ha sido el estandarte de la cirugía bariatrica es la manga gastrica laparoscopica. Este es un procedimiento restrictivo y que además tiene un fuerte componente hormonal ya que logra disminuir ostensiblemente los niveles de ghrelina, una hormona relacionada con el complejo mecanismo del apetito-saciedad, trayendo como consecuencia clínica una disminución en el apetito(Makarem-Kanso, Miquilarena, Navas, Fermín, \& Jiménez, 2008).

Por lo tanto, el objetivo general de esta investigación es analizar las complicaciones frecuentes inmediatas en pacientes de 20-29 años sometidos a manga gástrica laparoscópica del Hospital Teodoro Maldonado. La metodología empleada es a través de investigaciones de tipo documental y bibliográfica.

\section{Método.}

Se realizó una metodología de tipo documental y bibliográfica gracias a los análisis de la obesidad en la sociedad, los diferentes tratamientos que se estipulan para esta enfermedad de la era globalizada, la manga gástrica laparoscópica como alternativa actual para la obesidad mórbida y las complicaciones postoperatorias de esta cirugía. Todo esto gracias a las herramientas de investigación como textos, documentos y artículos científicos publicados disponibles en la web. 


\section{Complicaciones frecuentes inmediatas en paciente de 20-29 años sometidos a manga gástrica laparoscópica del Hospital Teodoro Maldonado}

Vol. 3, núm. 3., (2019)

Ana María Viteri Rojas; Katherine Angélica Sánchez Alcantara; Andrea Alexandra Saltos

Román

\section{Resultados.}

Características de la cirugía de manga gástrica laparoscópica

Una de las técnicas de cirugía bariátrica es la manga gástrica laparoscópica (MGL). Esta técnica es joven en comparación de otras de mayor data. Fueron Baltasar y col. los primeros en proponer a la MGL como procedimiento bariátrico único, ya que observaron que en muchos casos no se necesitaba recurrir a una segunda fase quirúrgica porquese conseguía una disminución de peso importante(González, Lepe, López Espinosa, \& Chávez, 2014).La manga gástrica es un procedimiento que no produce malabsorción, mantiene íntegra la continuidad intestinal y no requiere dispositivos exógenos que ameriten ajustarse(Makarem-Kanso, Miquilarena, Navas, Fermín, \& Jiménez, 2008).

La MGL se realiza una resección longitudinal del estómago, paralela a la curvatura mayor, iniciando a 3-5 $\mathrm{cm}$ del píloro ascendiendo hacia la incisura angularis y dejando un remanente gástrico de tipo tubular con preservación el píloro(Acquafresca, Palermo, Duza, Blanco, \& Serra, 2015). Esta técnica se puede detallar en la Figura 2.Esta técnica nueva posee factores que involucran su éxito en la reducción de peso rápido en los pacientes de obesidad mordica debido a la reducción de la producción de ghrelina. 


\section{Complicaciones frecuentes inmediatas en paciente de 20-29 años sometidos a manga gástrica laparoscópica del Hospital Teodoro Maldonado}

Vol. 3, núm. 3., (2019)

Ana María Viteri Rojas; Katherine Angélica Sánchez Alcantara; Andrea Alexandra Saltos Román

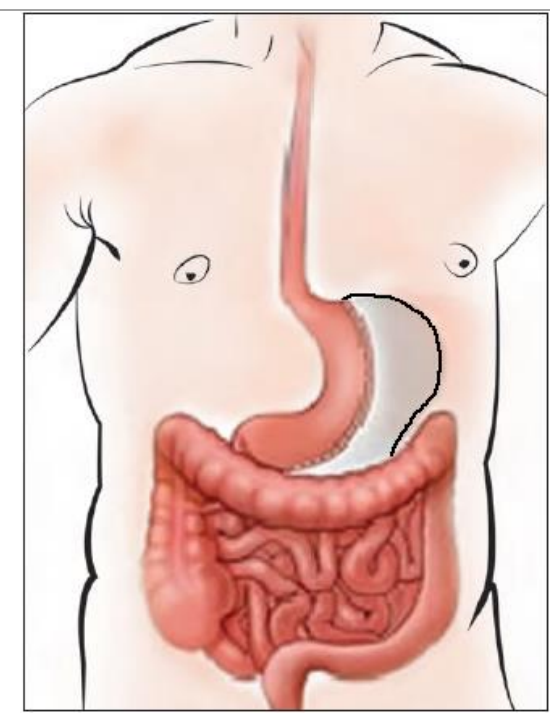

Figura 2. Objetivo de la manga gástrica laparoscópica

Fuente: (Acquafresca, Palermo, Duza, Blanco, \& Serra, 2015)

“El primero es la reducción de la capacidad volumétrica del estómago, la cual puede variar según la bujía de calibración y donde se inicie la resección gástrica tomando como punto de referencia el píloro. Un segundo factor es la prevalencia del píloro que podría funcionar como una banda gástrica natural contribuyendo al efecto de la saciedad”. (Nassar, 2011, pág. 266)

Estos factores no alteran la fisiología del estómago. Es una operacióntécnicamente más simple que el Bypass gástrico, no se presentaningún síndrome de vaciamiento rápido porque sepreserva el píloro, no se dejan cuerpos extraños ni aditivosespeciales en el interior y la recuperación del paciente esmuy rápida(Marcuschamer, y otros, 2009). Además, el remanente gástrico es accesible vía endoscópica, lo cual permite detectar patologías gástricas como sería un 


\section{Complicaciones frecuentes inmediatas en paciente de 20-29 años sometidos a manga gástrica laparoscópica del Hospital Teodoro Maldonado}

Vol. 3, núm. 3., (2019)

Ana María Viteri Rojas; Katherine Angélica Sánchez Alcantara; Andrea Alexandra Saltos

Román

cáncer gástrico; así mismo posee desventajas conocidas como mayor índice de reflujo gastroesofágico postoperatorio y mayor índice de fugas(Rodríguez-Esquerra \& BeltránGastélum, 2013).

Las indicaciones para realizar esta operación son diversas y dependen del diagnóstico realizado por el especialista. Depende del grado de obesidad y de problemas colaterales que conlleva el sobrepeso. Esta técnica quirúrgica puede ser empleada como cirugía única o como cirugía "en dos tiempos" con la finalidad de reducción de peso y riesgo cardiovascular para poder realizar una cirugía más compleja o más "definitiva”(Morales-Maza, 2018).Además, el índice de masa corporal debe ser mayor a (IMC) $30-35 \mathrm{~kg} / \mathrm{m} 2$ con la justificación de comorbilidades ya determinadas (diabetes mellitus [DM], hipertensión arterial sistémica, dislipidemias, síndrome de apnea obstructiva del sueño, cáncer, etc.)(González, Lepe, López Espinosa, \& Chávez, 2014).

El inicio de la cirugía empieza con la colocación de la anestesia en el paciente. Luego, se coloca en decúbito ventral con la cabeza elevada30 grados (Trendelemburg invertido) y ligera rotación a la izquierda, para luego colocar cinco trócares en la parte superior del abdomen y utilizamos óptica de treinta grados(Makarem-Kanso, Miquilarena, Navas, Fermín, \& Jiménez, 2008). Esto se puede observar a través de la Figura 3. 


\section{Complicaciones frecuentes inmediatas en paciente de 20-29 años sometidos a manga gástrica laparoscópica del Hospital Teodoro Maldonado}

Vol. 3, núm. 3., (2019)

Ana María Viteri Rojas; Katherine Angélica Sánchez Alcantara; Andrea Alexandra Saltos Román

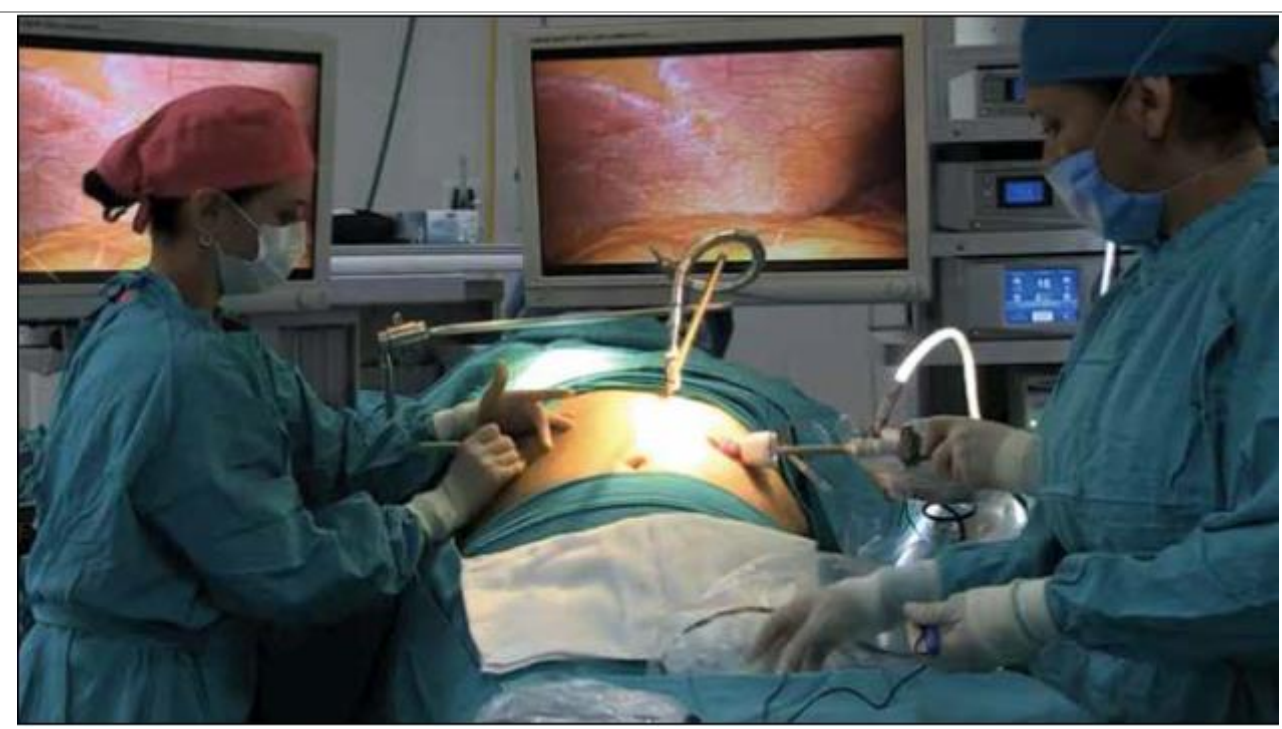

Figura 3. Inserción de trocares en paciente

Fuente: (González, Lepe, López Espinosa, \& Chávez, 2014)

Seguidamente se introduce una cámara de 30 grados para una inspección dela cavidad abdominal. Se realiza tracciónhepática y exposición de hiatoesofágico, luego se realiza la disección del ligamentogastrocolico con bisturíultrasónico iniciando a $5-7 \mathrm{~cm}$ de la válvulapilórica para posteriormente entrara la retrocavidad de los epiplones(González, Lepe, López Espinosa, \& Chávez, 2014), tal cual se puede visualizar en la Figura 4. 


\section{Complicaciones frecuentes inmediatas en paciente de 20-29 años sometidos a manga gástrica laparoscópica del Hospital Teodoro Maldonado}

Vol. 3, núm. 3., (2019)

Ana María Viteri Rojas; Katherine Angélica Sánchez Alcantara; Andrea Alexandra Saltos

Román

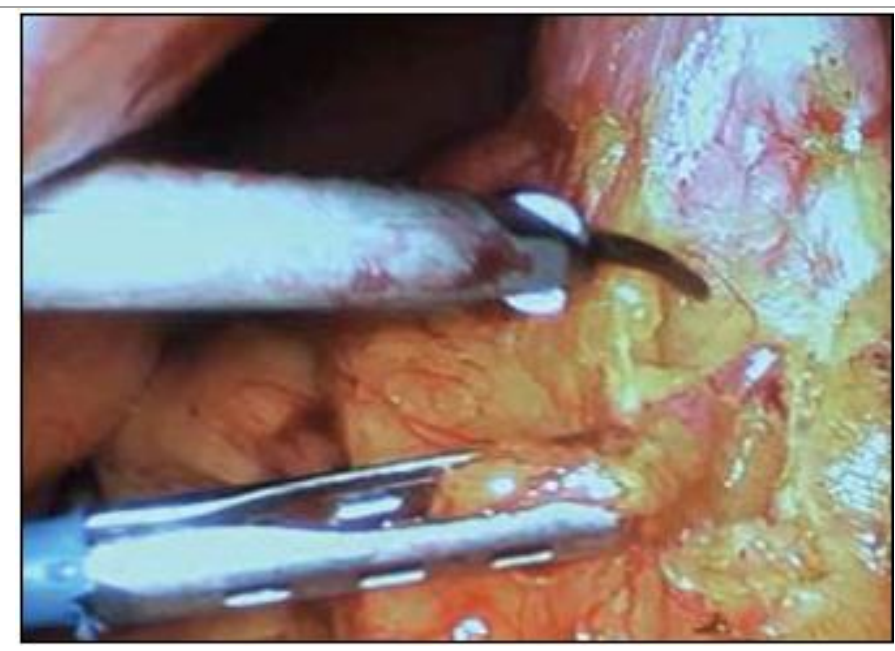

Figura 3. Disección con bisturí ultrasonico en el ligamento gastrocolico

Fuente: (González, Lepe, López Espinosa, \& Chávez, 2014)

Después se introduce una bujía esofágica calibre $36 \mathrm{Fr}$ por medio de la boca y ayudado por el laparoscopio hasta llegar al píloro. Utilizando laparoscópica de autosutura con recarga verde de $60 \mathrm{~mm}$ triple hilera grapas y guiados por la bujía se comienza la gastrectomía desde el antro a $4 \mathrm{~cm}$ del píloro, luego recargas azules hasta el ángulo de His alejándose $1 \mathrm{~cm}$ del esófago en el último disparo(Makarem-Kanso, Miquilarena, Navas, Fermín, \& Jiménez, 2008). Esto se puede mostrar en la Figura 5. 


\section{Complicaciones frecuentes inmediatas en paciente de 20-29 años sometidos a manga gástrica laparoscópica del Hospital Teodoro Maldonado}

Vol. 3, núm. 3., (2019)

Ana María Viteri Rojas; Katherine Angélica Sánchez Alcantara; Andrea Alexandra Saltos Román

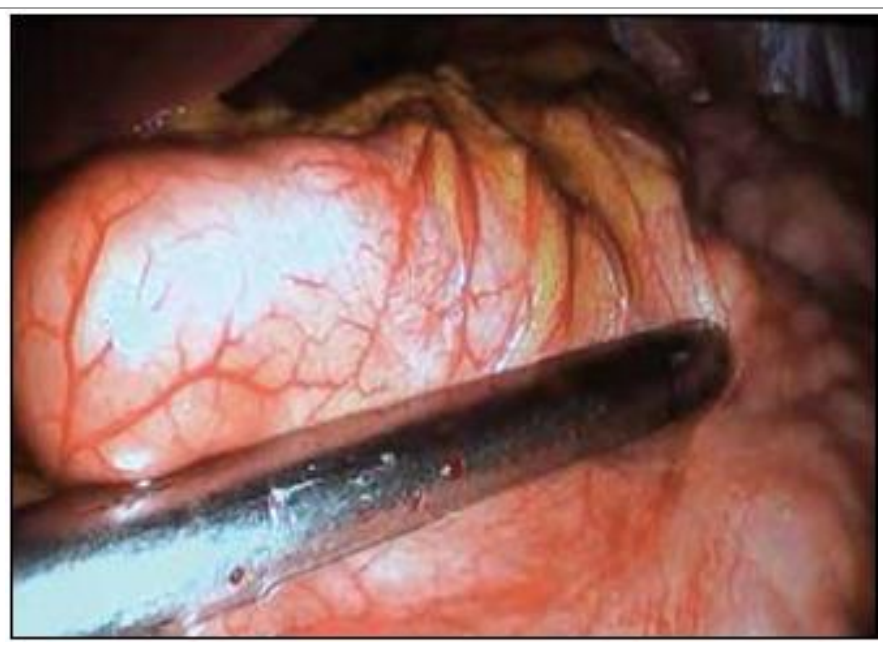

Figura 5. Colocación de grapas para crear el neoestómago

Fuente: (González, Lepe, López Espinosa, \& Chávez, 2014)

Se realiza examen hidroneumático en el cual se introducen $500 \mathrm{ml}$ de solución salina hasta cubrir por completo el neoestómago. Se colocauna pinza de oclusión distal a la línea de grapadopara insuflary poder observar burbujeo, después se instila azul de metilenoa través de la sonda, todo esto para corroborarhermeticidad del neoestómago (González, Lepe, López Espinosa, \& Chávez, 2014), como se puede observar en la Figura 6. 


\section{Complicaciones frecuentes inmediatas en paciente de 20-29 años sometidos a manga gástrica laparoscópica del Hospital Teodoro Maldonado}

Vol. 3, núm. 3., (2019)

Ana María Viteri Rojas; Katherine Angélica Sánchez Alcantara; Andrea Alexandra Saltos

Román

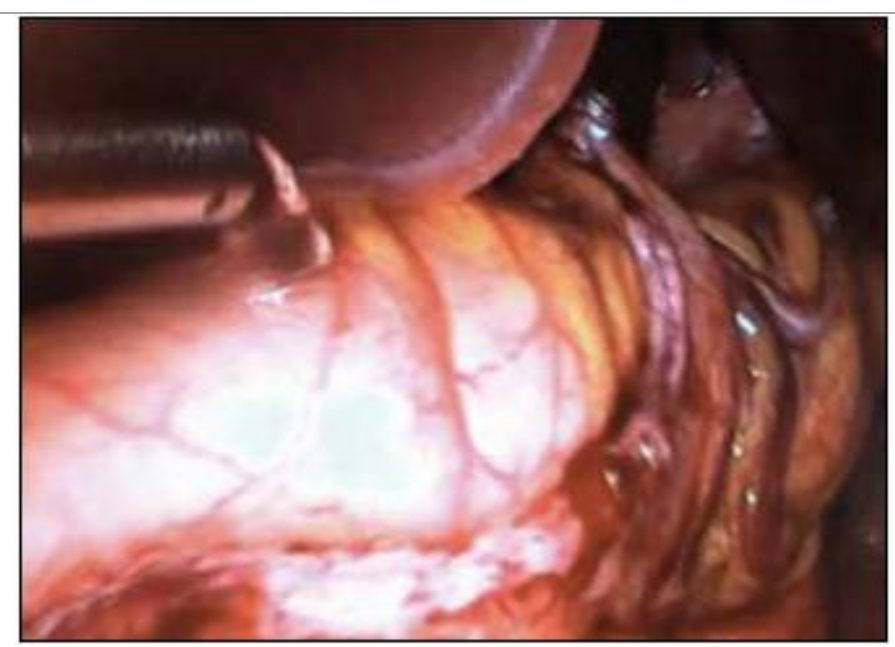

Figura 6. Ensayo para determinar la hermeticidad del neoestómago con el fin de evitar fugas en las grapas o suturas

Fuente: (González, Lepe, López Espinosa, \& Chávez, 2014)

Se retira la retracción hepática. Se coloca y fija un drenaje Jackson-PrattR (Cardinal Health, McGaw Park, IL, USA, 19 Fr) con nylon de 2-0, y se sutura el tejidocelular subcutáneo con catgut simple de 2-0, finalmente se sutura con monocryl de 3-0 puntos subdérmicos para la piel (González, Lepe, López Espinosa, \& Chávez, 2014).La mayoría de los pacientes no se drenan, pasan a recuperación y luego a la habitación(Makarem-Kanso, Miquilarena, Navas, Fermín, \& Jiménez, 2008).

La perdida de peso rápida después de la cirugía de MGL es por la hormona ghrelina. Esta proviene del hipotálamo y va dirigida al estómago. El hipotálamo es la región del sistema nervioso central que sirve de regulador central del apetito y la distribución grasa del cuerpo,esto 


\section{Complicaciones frecuentes inmediatas en paciente de 20-29 años sometidos a manga gástrica laparoscópica del Hospital Teodoro Maldonado}

Vol. 3, núm. 3., (2019)

Ana María Viteri Rojas; Katherine Angélica Sánchez Alcantara; Andrea Alexandra Saltos Román

producto de dos neuronas: las que producen apetito como las orexigénicas y las que lo inhibe como las anorexigénicas(Corvalá, y otros, 2008).

Estas dos vías son las que estudian el comportamiento de la obesidad en los pacientes y por supuesto intervienen en el balance energético en el ser humano. El comportamiento de la ghrelina es el catalizador para el aumento de peso desde el punto de vista fisiológico. Estas vías se pueden detallar en la Figura 7.

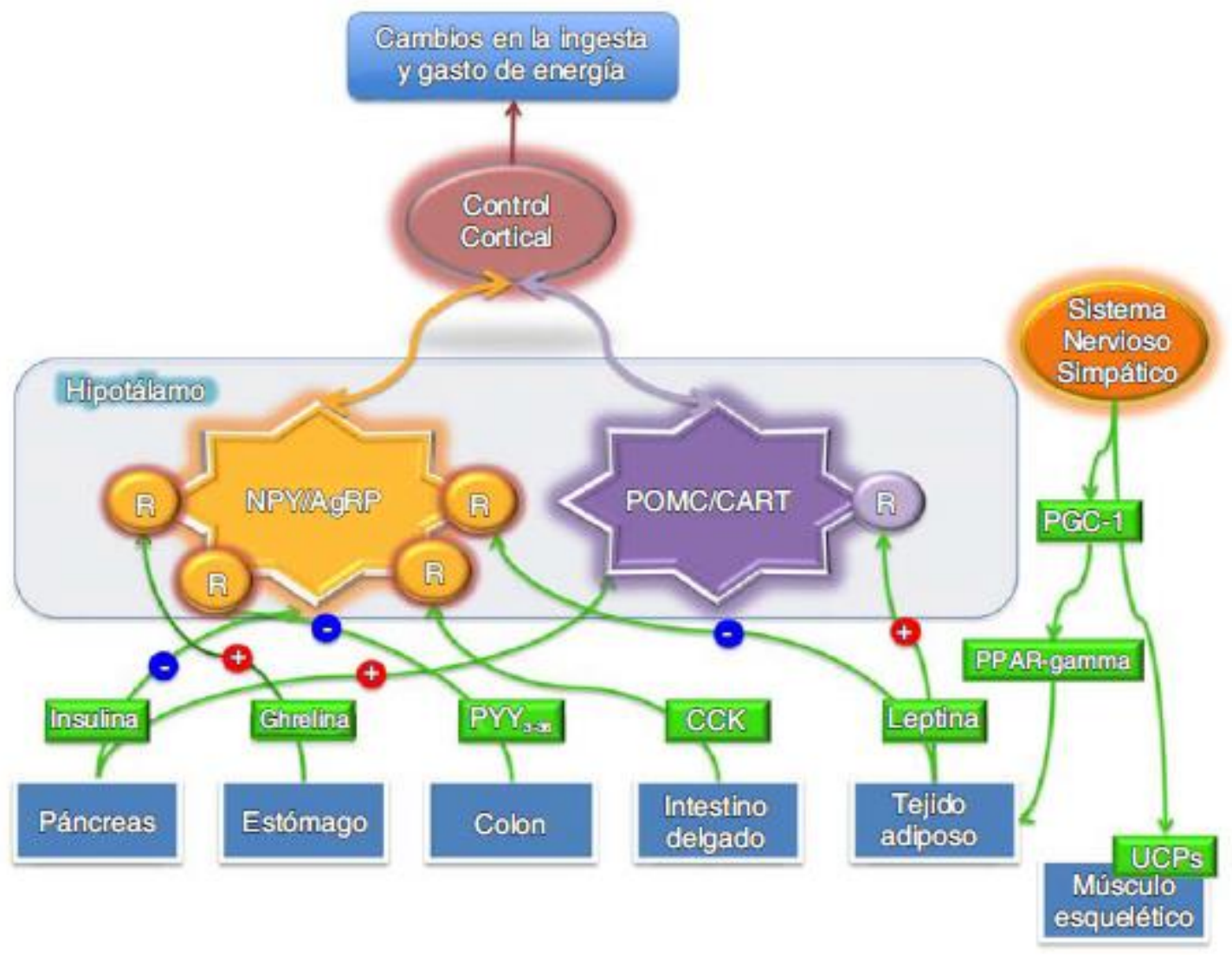

Figura 7. Vías de regulación del control en la homeostasis de energía

Fuente: (Corvalá, y otros, 2008) 


\section{Complicaciones frecuentes inmediatas en paciente de 20-29 años sometidos a manga gástrica laparoscópica del Hospital Teodoro Maldonado}

Vol. 3, núm. 3., (2019)

Ana María Viteri Rojas; Katherine Angélica Sánchez Alcantara; Andrea Alexandra Saltos

Román

Existe otro mecanismo por la cual existe disminución de peso a través de la MGL y es la reducción de volumen de la cámara gástrica. Se han realizado varios estudiosdonde se ha demostrado que la distensióngástrica produce una sensación de saciedad en humanos, donde se inicia una cascada de activación sináptica que origina desde baroreceptores gástricos hacia el núcleoentremediar viajando por axonesdel nervio vago(González, Lepe, López Espinosa, \& Chávez, 2014).

\section{Complicaciones de la MGL}

Los tratamientos quirúrgicos para la obesidad no escapan de sufrir complicaciones. Según las estadísticas las complicaciones son mayores en el Bypass gástrico (BGYR) que la de manga gástrica debido a la complejidad de la cirugía. El BGYR implica la seccióndel estómago y del intestino delgado y la creación de dosanastomosis, mientras que en la MGL no hay anastomosisde por medio, y solo una gran línea de sutura a lo largo delárea de sección gástrica(Acquafresca, Palermo, Duza, Blanco, \& Serra, 2015).

Para la MGL existen complicaciones en función del tiempo postoperatorio. Una son las complicaciones tempranas y otras son las complicaciones tardías. En los abordajes laparoscópicos se reporta una incidencia del 5 al 13\%(Bolaños \& Porras, 2014). En pacientes super obesos (IMC 50-60 kg/m2) la tasa de complicaciones parece incrementar de manera importante, debido a la dificultad de abordar quirúrgicamente por su gran tamaño y el grado de comorbilidades que presentan al momento de ser intervenidos(González, Lepe, López Espinosa, \& Chávez, 2014).En la Tabla 3 se detalla las complicaciones tempranas y en la Tabla 4 se observa las complicaciones tardías más frecuentes. 


\section{Complicaciones frecuentes inmediatas en paciente de 20-29 años sometidos a manga gástrica laparoscópica del Hospital Teodoro Maldonado}

Vol. 3, núm. 3., (2019)

Ana María Viteri Rojas; Katherine Angélica Sánchez Alcantara; Andrea Alexandra Saltos

Román

\section{Tabla 3.}

Complicaciones tempranas de la cirugía por manga gástrica laparoscópica.

\begin{tabular}{|c|c|}
\hline Complicaciones & Características \\
\hline \multirow{5}{*}{ Dehiscencia } & Cualquier sutura del tubo digestivo puede fracasar. \\
\hline & El tipo de dehiscencia está en relación con el tipo de operación efectuada. \\
\hline & $\begin{array}{l}\text { Se mantienen algunos preceptos tradicionales: son más graves cuanto más } \\
\text { precoces y cuanto más proximales, también son más graves las laterales que } \\
\text { las terminales (muñón duodenal) o de asas aisladas (Y de Roux). }\end{array}$ \\
\hline & $\begin{array}{l}\text { Siempre son complicaciones graves, van seguidas de una peritonitis, con sus } \\
\text { secuelas de colecciones abdominales y sepsis, se manifiestan como fístulas } \\
\text { externas cuyo débito estará en relación con el tamaño de la dehiscencia y la } \\
\text { permeabilidad del tubo digestivo distal. }\end{array}$ \\
\hline & $\begin{array}{l}\text { Se las divide en de alto y bajo débito, de acuerdo a si éste supera o no los } 500 \\
\mathrm{ml} / 24 \mathrm{hs}\end{array}$ \\
\hline \multirow{8}{*}{ Fístulas gástricas } & Generalmente en los primeros 7 días postoperatorios \\
\hline & $\begin{array}{l}\text { De las fístulas gástricas se reporta que } 89 \% \text { ocurren en el tercio superior de la } \\
\text { línea de grapas colocada, cerca del ángulo de His }\end{array}$ \\
\hline & $\begin{array}{l}\text { Se puede presentar clínicamente como dolor abdominal, ataque al estado } \\
\text { general, fiebre y evolucionar a complicaciones más graves como síndrome } \\
\text { séptico. }\end{array}$ \\
\hline & $\begin{array}{l}\text { Cuando la pérdida de líquido intestinal se difunde y se acumula libremente } \\
\text { dentro del abdomen, desarrollará rápidamente una peritonitis. }\end{array}$ \\
\hline & $\begin{array}{l}\text { Si la salida del líquido intestinal no tiene escape al exterior y es de escasa } \\
\text { magnitud quedará atrapada en los distintos espacios intraperitoneales } \\
\text { conformando uno o más abscesos. }\end{array}$ \\
\hline & $\begin{array}{l}\text { Finalmente, cuando el material entérico aflora en la piel se establece una } \\
\text { fístula enterocutánea. Se observa entonces, que la fístula enterocutánea tiene } \\
\text { un origen común con otras complicaciones relacionadas con la perforación } \\
\text { intestinal, y puede coexistir con ellas. }\end{array}$ \\
\hline & $\begin{array}{l}\text { En estos pacientes cualquier colección fuera de la línea de grapas se debe } \\
\text { considerar absceso secundario a fístula gástrica, especialmente si contiene gas. }\end{array}$ \\
\hline & $\begin{array}{l}\text { El manejo no quirúrgico con drenaje percutáneo guiado radiológicamente } \\
\text { reporta un éxito de hasta el } 86 \% \text {. }\end{array}$ \\
\hline \multirow{3}{*}{ Trombosis portal } & $\begin{array}{l}\text { Esta complicación debe sospecharse en los pacientes en quienes se descarta la } \\
\text { fístula gástrica y presentan dolor abdominal, síndrome de respuesta } \\
\text { inflamatoria sistémica y esplenomegalia. }\end{array}$ \\
\hline & $\begin{array}{l}\text { Los estudios confirmatorios son la TAC con contraste EV (endovenosa) y el } \\
\text { doppler portal. }\end{array}$ \\
\hline & El tratamiento es la anticoagulación \\
\hline \multirow{3}{*}{$\begin{array}{l}\text { Hemorragia gástrica } \\
\text { intraluminal }\end{array}$} & En estos casos se valora la estabilidad hemodinámica del paciente. \\
\hline & $\begin{array}{l}\text { Si no provoca descompensación hemodinámica se recomienda conducta } \\
\text { expectante. }\end{array}$ \\
\hline & $\begin{array}{l}\text { una vez descartada la hemorragia intraabdominal, se realizará endoscopía } \\
\text { diagnóstica y/o terapéutica }\end{array}$ \\
\hline \multirow{3}{*}{$\begin{array}{l}\text { Hemorragia gástrica } \\
\text { intraabdominal }\end{array}$} & Su resolución es quirúrgica. \\
\hline & $\begin{array}{l}\text { De presentarse hematomas intraabdominales que requieran ser drenados se } \\
\text { recomienda conservar el drenaje durante un tiempo prolongado ya que } \\
\text { frecuentemente estos son colecciones secundarias a fístulas gástricas. }\end{array}$ \\
\hline & $\begin{array}{l}\text { La presencia de palidez, mareos, confusión, taquicardia, hipotensión, oliguria, } \\
\text { hematemesis y/o hematoquecia, debe fomentar la sospecha clínica de esta }\end{array}$ \\
\hline
\end{tabular}




\title{
Complicaciones frecuentes inmediatas en paciente de 20-29 años sometidos a manga gástrica laparoscópica del Hospital Teodoro Maldonado
}

\author{
Ana María Viteri Rojas; Katherine Angélica Sánchez Alcantara; Andrea Alexandra Saltos \\ Vol. 3, núm. 3., (2019)
}

Román

complicación, para su abordaje de manera oportuna

Fuente: (Bolaños \& Porras, 2014; Vizcaíno Bautista, 2016)

Tabla 4.

Complicaciones tardías de la cirugía por manga gástrica laparoscópica.

Complicaciones

Características

Corresponde con la disminución en el calibre del tubo gástrico no modificado

en los estudios radiológicos dinámicos sumado a disfagia, vómitos, náuseas y

reflujo de Novo; los cuales no permiten una alimentación correcta.

Su incidencia se ha reportado de 0.26 a $4 \%$.

El sito de presentación más frecuente es la cisura angularis y se ha sugerido que la causa es una deficiencia técnica por la angulación incorrecta de la engrapadora o bien por el uso de una sonda de calibración grande asociada a una retracción excesiva de la curvatura mayor durante el primer corte.

Factores intrínsecos en la anatomía del paciente constituyen otro factor causal, al haber una rotación gástrica en espiral dando lugar a que la línea de sutura se presente en un plano anterior a posterior, lo cual, a pesar de que la manga tenga un calibre adecuado puede generar síntomas obstructivos

Estenosis de tubo gástrico

La sintomatología suele iniciar de 6 a 24 meses post operatorios

Los estudios radiológicos permiten confirmar el diagnóstico y establecer la conducta terapéutica dependiendo de la localización y longitud de la estenosis.

En los casos en donde la estenosis es corta se recomienda la dilatación con balón, en una o varias sesiones. Por el contrario, si la estenosis es larga la dilatación tiene alta tasa de fracaso por lo que se recomienda la seromiotomía, la cual se puede realizar con abordaje laparoscópico, con o sin control endoscópico simultáneo.

Es importante que durante este procedimiento se realice un control con instilación de solución fisiológica con azul de metileno y la insuflación con aire bajo agua posteriormente, debido al riesgo de perforación de la mucosa, ya que en los casos no identificados ocasionaría una fístula gástrica.

Si a pesar de estos tratamientos la sintomatología continúa, se debe convertir a otra cirugía bariátrica.

Falla del mecanismo antirreflujo permitiendo el reflujo anormal del contenido gástrico al esófago

Se presenta con una incidencia del 1 al 36\% en el primer año postquirúrgico.

Posterior al tercer año postoperatorio la incidencia disminuye

Usualmente se asocia con a descenso en la pérdida de peso, dilatación del "pouch" y recuperación del ángulo de His.

En estos pacientes la sensación de pirosis o bien regurgitación asociada a documentación endoscópica, ph-metría de 24 horas o esofagograma, son diagnósticos de esta complicación

Reflujo gastroesofágico Puede ser causado por disminución de la presión del esfínter esofágico inferior debido a la sección de las fibras musculares tanto longitudinales como oblicuas, aumento de la presión en la cavidad intragástrica, sección extrema del antro con desaparición del ángulo de His o dilatación de la parte superior de la manga

Debido a que el RGE (Reflujo Gastroesofágico) puede presentarse en el PO inmediato y mediato se sugiere la utilización de inhibidores de bomba de protones durante los primeros 3 meses del postoperatorio como tratamiento.

De inicio siempre tratamiento médico con inhibidores de la bomba de protones asociados con proquinéticos y reeducación alimentaria. 


\section{Complicaciones frecuentes inmediatas en paciente de 20-29 años sometidos a manga gástrica laparoscópica del Hospital Teodoro Maldonado}

Vol. 3, núm. 3., (2019)

Ana María Viteri Rojas; Katherine Angélica Sánchez Alcantara; Andrea Alexandra Saltos Román

\begin{tabular}{|c|c|}
\hline & $\begin{array}{l}\text { De ser resistente al tratamiento puede plantearse la conversión de manga a } \\
\text { bypass gástrico laparoscópico. }\end{array}$ \\
\hline \multirow{5}{*}{ Dilatación gástrica } & $\begin{array}{l}\text { La dilatación gástrica o dilatación del pouch, luego de gastrectomía, en manga } \\
\text { es un fenómeno descrito con distintos porcentajes de incidencia no } \\
\text { homogenizados aún. }\end{array}$ \\
\hline & $\begin{array}{l}\text { Su manifestación principal es una re ganancia de peso o bien una pérdida de } \\
\text { peso insatisfactoria ( }<50 \% \text { exceso de peso a los } 18 \text { meses }) \text {; aunque hay } \\
\text { reportes que han documentado dilatación gástrica por tomografía sin presentar } \\
\text { manifestaciones clínicas }\end{array}$ \\
\hline & $\begin{array}{l}\text { La dilatación del pouch generalmente se asocia a la presión excesiva } \\
\text { intraluminal debido a grandes porciones de alimentos, vómitos frecuentes o } \\
\text { bien obstrucción distal. También en los casos de pacientes con hernia hiatal } \\
\text { que, sumada a la obesidad extrema, dificulta la disección del fondo gástrico. }\end{array}$ \\
\hline & $\begin{array}{l}\text { El aumento en la presión intra gástrica de un aproximado de } 26 \mathrm{~mm} \mathrm{Hg} \text { pre } \\
\text { operatoria a } 43 \mathrm{~mm} \mathrm{Hg} \mathrm{luego} \mathrm{de} \mathrm{la} \mathrm{cirugía,} \mathrm{relacionado} \mathrm{con} \mathrm{la} \mathrm{disminución} \mathrm{del} \\
\text { tamaño del estómago es otro factor que se ha asociado a aumento de } \\
\text { distensibilidad progresiva de la pared. }\end{array}$ \\
\hline & $\begin{array}{l}\text { Se proponen diferentes formas de manejo de esta complicación, algunos } \\
\text { autores sugieren la conversión a otros procedimientos como bypass gástrico o } \\
\text { derivación bilio digestiva, mientras otros prefieren una re gastrectomía en } \\
\text { manga, no existiendo consenso al respecto }\end{array}$ \\
\hline \multirow{4}{*}{$\begin{array}{l}\text { Deficiencias } \\
\text { nutricionales }\end{array}$} & $\begin{array}{l}\text { La gastrectomía en manga como procedimiento restrictivo puede generar } \\
\text { deficiencias en ciertos componentes nutricionales y manifestarse clínicamente. }\end{array}$ \\
\hline & $\begin{array}{l}\text { Esto es debido a la reducción substancial de la ingesta calórica, la disminución } \\
\text { del volumen de secreción de ácido clorhídrico y factor intrínseco, la potencial } \\
\text { aparición de náuseas y vómitos, la intolerancia a ciertos alimentos sólidos con } \\
\text { mejor calidad nutricional, entre otros. }\end{array}$ \\
\hline & $\begin{array}{l}\text { Recientemente se reportó que en pacientes post operados de gastrectomía en } \\
\text { manga puede encontrarse una disminución de vitaminas y micronutrientes } \\
\text { como Vitamina B } 12 \text { en } 3 \% \text {, Vitamina D en } 23 \% \text {, folato } 3 \% \text {, hierro } 3 \% \text {, y Zinc } \\
\text { en } 14 \% \text {. }\end{array}$ \\
\hline & $\begin{array}{l}\text { Es recomendable por tanto realizar controles seriados de niveles de estos } \\
\text { componentes al menos } 3,6 \text { y } 12 \text { meses luego de la cirugía con el fin de tratar } \\
\text { precozmente cualquier deficiencia }\end{array}$ \\
\hline
\end{tabular}

Fuente: (Bolaños \& Porras, 2014; Vizcaíno Bautista, 2016; (Us de Paz \& Contreras Parraguez, 2015))

La cirugía de MGL, en adultos jóvenes, en el Hospital Teodoro Maldonado

Para el segundo semestre del año 2013, se realizaron 115 cirugías de las cuales 46

pertenecen a adultos jóvenes (40\% de la muestra estudiada), donde el $68,7 \%$ proviene de la provincia de Guayas en Ecuador (PARRA VILLEGAS, 2015). Los pacientes presentan una patología antes del ingreso al hospital y por ende antes de la cirugía de MGL que se muestra en 


\section{Complicaciones frecuentes inmediatas en paciente de 20-29 años sometidos a manga gástrica laparoscópica del Hospital Teodoro Maldonado}

Vol. 3, núm. 3., (2019)

Ana María Viteri Rojas; Katherine Angélica Sánchez Alcantara; Andrea Alexandra Saltos

Román

la Figura 8. Cabe destacar que estos porcentajes es en función de los 115 pacientes tratados en el centro médico.

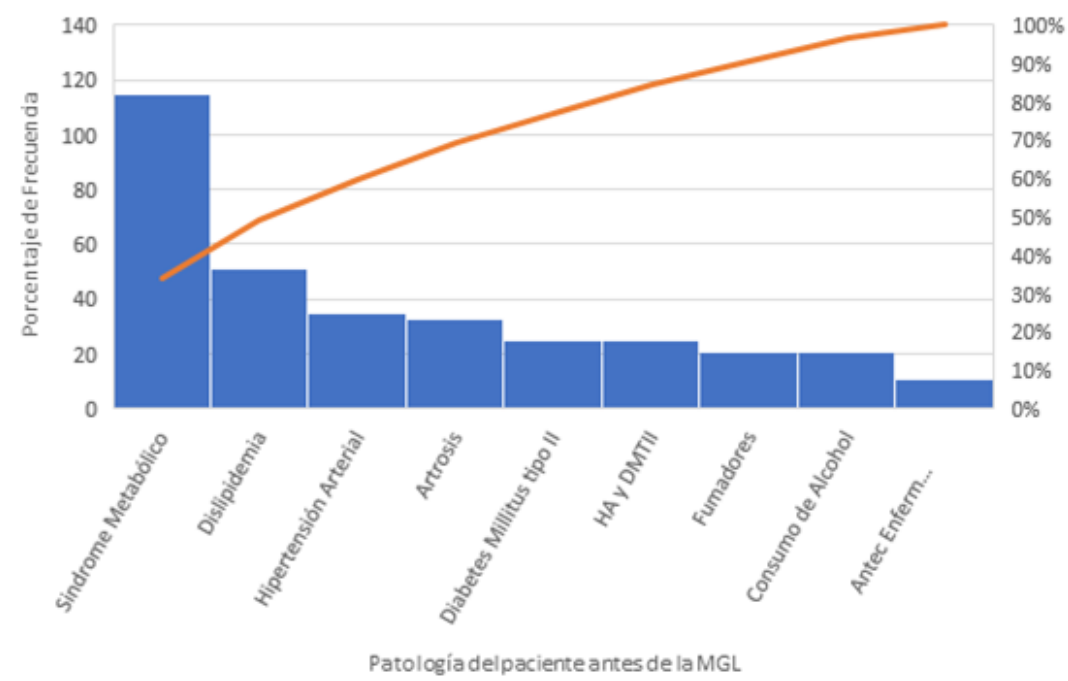

Figura 8. Patología de los 115 pacientes sometidos a MGL en el Hospital Teodoro Maldonado en el último semestre del 2013. Fuente: (PARRA VILLEGAS, 2015)

De estos 115 pacientes atendidos 21 tuvieron complicaciones postoperatorias de los cuales 8 son adultos jóvenes(PARRA VILLEGAS, 2015). Los tipos de complicaciones se detallan en la Figura 9. 


\section{Complicaciones frecuentes inmediatas en paciente de 20-29 años sometidos a manga gástrica laparoscópica del Hospital Teodoro Maldonado}

Vol. 3, núm. 3., (2019)

Ana María Viteri Rojas; Katherine Angélica Sánchez Alcantara; Andrea Alexandra Saltos Román

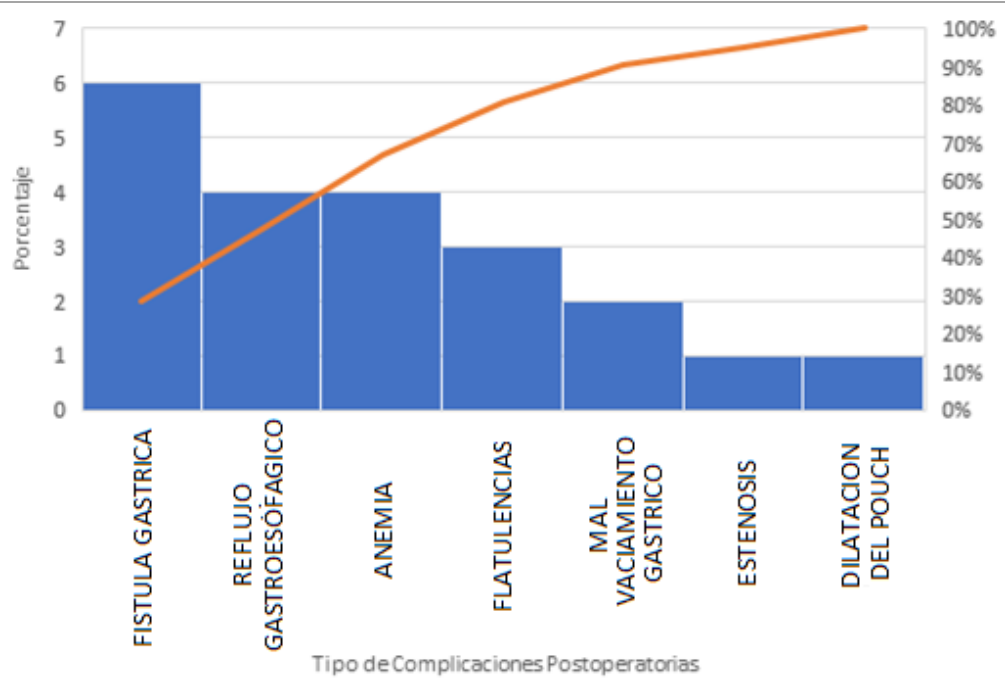

Figura 9. Tipos de complicaciones postoperatorias de 21 pacientes sometidos a MGL en el Hospital Teodoro Maldonado en el último semestre del 2013. Fuente: (PARRA VILLEGAS, 2015)

Estas complicaciones son debidas a múltiples factores. Estos son: la perdida en el seguimiento de los pacientes, mal control multidisciplinario postoperatorio, no cumplimiento por parte de los pacientes de las recomendaciones médicas(PARRA VILLEGAS, 2015). Existe una relación entre la complicación y la patología del paciente antes de la cirugía de MGL donde esta última influye en las consecuencias postoperatorias. Esta se puede visualizar en la Figura 10. 


\section{Complicaciones frecuentes inmediatas en paciente de 20-29 años sometidos a manga gástrica laparoscópica del Hospital Teodoro Maldonado}

Vol. 3, núm. 3., (2019)

Ana María Viteri Rojas; Katherine Angélica Sánchez Alcantara; Andrea Alexandra Saltos

Román

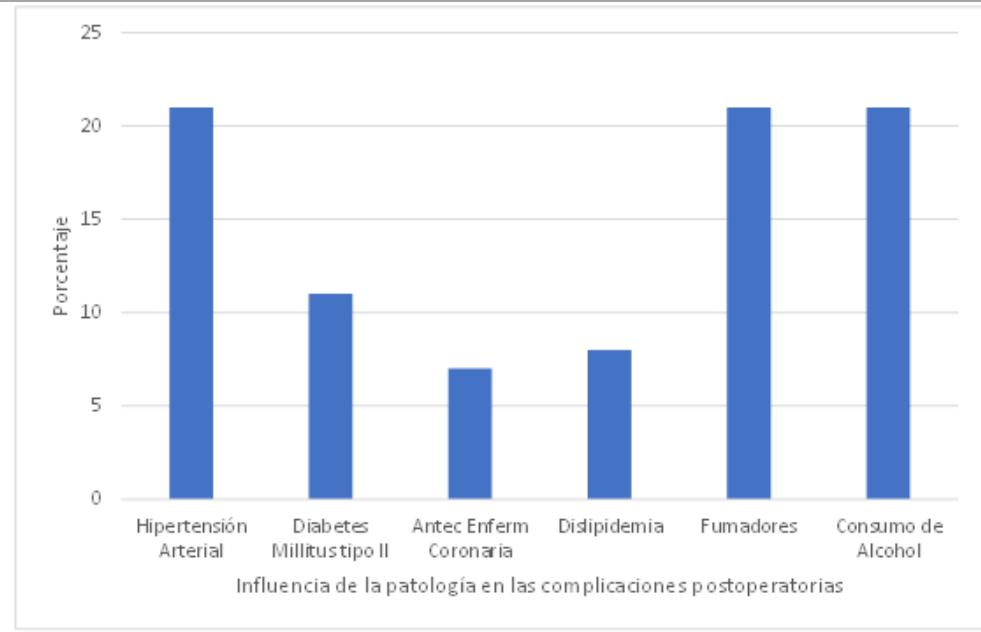

Figura 10. Influencia de la patología de los 21 pacientes con complicaciones postoperatorias sometidos a MGL en el Hospital Teodoro Maldonado en el último semestre del 2013. Fuente: (PARRA VILLEGAS, 2015)

\section{Discusión y conclusiones}

Uno de las consecuencias que trae la globalización y la dependencia excesiva de la tecnología es la obesidad en la población. Esto es debido a que a mayor crecimiento de la sociedad menor es el tiempo que se genera para poder realizar ejercicios físicos y comidas balanceadas produciendo sedentarismo e ingesta de alimentos con altos porcentajes de grasa y azúcares. La obesidad es una de las enfermedades más comunes y tienen múltiples factores que la originan. Desde el punto de vista fisiológico, el hipotálamo cumple la función de generar la ingesta o no de los alimentos gracias a que pertenece al sistema nervioso central, a su vez puede generar las diversas hormonas que permiten el equilibrio energético del cuerpo humano. Entre 


\section{Complicaciones frecuentes inmediatas en paciente de 20-29 años sometidos a manga gástrica laparoscópica del Hospital Teodoro Maldonado}

Vol. 3, núm. 3., (2019)

Ana María Viteri Rojas; Katherine Angélica Sánchez Alcantara; Andrea Alexandra Saltos Román

esas hormonas están las ghrelinas que son enviadas al estómago y son las encargadas de aumentar la masa corporal en el individuo cuando esta aumenta de volumen. Al aumentar de peso, aumentan también otras enfermedades por efecto colateral, tales como problemas cardiacos, hipertensión arterial y diabetes mellitus. Existen muchos tratamientos que pueden ayudar a disminuir de peso, pero todas empiezan con la fuerza de voluntad que está ligada a la autoestima y deseo de superación, es decir un tratamiento cognitivo-psicológicos. Luego el cambio de dieta alimentaria par después seguir con ejercicios físicos.

Otro de los tratamientos son los quirúrgicos previa evaluación y autorización del especialista. Estos ocurren porque las enfermedades colaterales producen una disminución de la calidad de vida de los pacientes y es necesario recurrir a una disminución de peso considerable ya que los otros tratamientos no han podido cumplir dicha función. La cirugía bariátrica es el tratamiento por excelencia para el trato de la obesidad. La característica principal es que cumple con la función de disminuir el consumo de alimentos a través de diversas técnicas donde las principales son el Bypass gástrico y la manga gástrica laparoscópica. La primera es una de las cirugías más realizadas y se basa en la creación de un puente entre la entrada del estomago y la Y de Raux, donde las secreciones biliopancreaticas se encuentran separadas del tracto alimentario.

La MGL es una de las técnicas más modernas y más utilizadas en el momento. Se basa en la disminución del estómago a través de la utilización de grapas e implementos laparoscópicos. Esta reducción permite que no se genere la ansiedad de ingerir los alimentos por lo que la disminución de peso es más rápida. El procedimiento se basa en corte con bisturí y la utilización de grapas en el estómago para que posteriormente se realice ensayos hidromecánicos con la 


\section{Complicaciones frecuentes inmediatas en paciente de 20-29 años sometidos a manga gástrica laparoscópica del Hospital Teodoro Maldonado}

Vol. 3, núm. 3., (2019)

Ana María Viteri Rojas; Katherine Angélica Sánchez Alcantara; Andrea Alexandra Saltos

Román

finalidad de que no existan fugas en la zona de corte y en las grapas. Los cuidados postoperatorios dependen de las condiciones del paciente, tanto medicas como psicológicas, si hay un desbalance en el cuidado entonces se producirá complicaciones que dependerán del tiempo de realizada la cirugía.Las complicaciones se dividen en dos partes, las tempranas y las tardías. En las tempranas prevalece la dehiscencia, la fistulas gástricas y las hemorragias gástricas. En las complicaciones tardías están la estenosis, reflujo gastroesofágico y dilatación gástrica como las más importantes.

Ahora, poder en el Hospital Teodoro Maldonado de la ciudad de Guayaquil, Ecuador presente una alta tasa de cirugías por MGL donde la población adulta joven es considerable. Para estas operaciones es necesario estudiar la patología de los pacientes con el fin de poder determinar la efectividad de la cirugía. Estos factores pueden influir drásticamente en el desarrollo postoperacional de los individuos. Los adultos jóvenes, sufren de complicaciones postoperatoria principalmente de fistula gástrica y reflujo gastroesofágico, donde la primera es por consecuencia depérdida de líquido intestinal la cual de difunde y se acumula formando una fistula en la piel que puede tender a peritonitis y la segunda es por la falla del mecanismo que regula el antirreflujo gástrico del esófago. Esta dificultad postoperatoria se incrementa si el paciente joven sufre de hipertensión arterial más si es consumidor de alcohol y cigarrillos.

\section{Bibliografía.}

Acquafresca, P. A., Palermo, M., Duza, G. E., Blanco, L. A., \& Serra, E. E. (2015). Bypass gástrico vs manga gástrica: comparación de resultados sobre diabetes tipo 2, descenso de peso y complicaciones. Revisión de ensayos clínicos controlados aleatorizados. Acta Gastroenterológica Latinoamericana, 45(2), 143-154. 


\section{Complicaciones frecuentes inmediatas en paciente de 20-29 años sometidos a manga gástrica laparoscópica del Hospital Teodoro Maldonado}

Vol. 3, núm. 3., (2019)

Ana María Viteri Rojas; Katherine Angélica Sánchez Alcantara; Andrea Alexandra Saltos Román

Arriagada Solar, M. I. (2015). Evaluación psicopatológica en obesidad y post cirugía bariátrica. Barcelona, España: Trabajo de Grado - Universidad Autónoma de Barcelona, Facultad de Medicina.

Barrientos-Pérez, M., \& Flores-Huerta, S. (2008). ¿ Es la obesidad un problema médico individual y social? Políticas públicas que se requieren para su prevención. Boletín Médico del Hospital Infantil de México, 65(6), 639-651.

Bersh, S. (2006). La obesidad: aspectos psicológicos y conductuales. Revista colombiana de psiquiatría, 35(4), 537-546.

Bolaños, M. D., \& Porras, A. M. (2014). Complicaciones de la Cirugía Bariátrica-Banda gástrica ajustable, gastrectomía en manga y bypass gástrico. Revista Clínica Escuela de Medicina UCR-HSJD, 4(4), 18-25.

Corvalá, L., Antonio, J., Guzmán Cordero, F., Pallanez, F. O., Valdez, C. H., Enríquez, C. C., \& Morales, J. T. (2008). Manga gástrica laparoscópica como procedimiento bariátrico único. Revista Mexicana de Cirugía Endoscópica, 9(4), 165-169.

Cruzat-Mandich, C., Díaz-Castrillón, F., García, A. T., \& Ulloa, V. J. (2018). Cirugía bariátrica en pacientes jóvenes:¿ Cómo evalúan el proceso? Revista chilena de nutrición, 45(1), 17 27.

Daza, C. H. (2002). La obesidad: un desorden metabólico de alto riesgo para la salud. Colombia Médica, 33(2), 72-80.

Fobi, M. A. (2010). El presente y futuro de la cirugía bariátrica. Revista chilena de cirugía, 62(1), 79-82.

García, E. (2004). ¿ Qué es la obesidad? Revista de endocrinologia y nutrición, 12(4), 88-90.

González, L. M., Lepe, M., López Espinosa, G., \& Chávez, J. T. (2014). Manga gástrica laparoscópica: Revisión detallada. Revista de la Facultad de Medicina UNAM, 57(6), 17 26.

Luque, G. T., García-Martos, M., Gutiérrez, C. V., \& Vallejo, N. G. (2010). Papel del ejercicio físico en la prevención y tratamiento de la obesidad en adultos. Retos. Nuevas tendencias en educación física, deporte y recreación, (18), 47-51.

Makarem-Kanso, Z., Miquilarena, R., Navas, H., Fermín, D., \& Jiménez, R. (2008). Manga Gástrica Laparoscópica, técnica para el tratamiento quirúrgico de la obesidad mórbida: experiencia en 70 casos. Revista Venezolana de Cirugía, 61(3), 125-130. 


\section{Complicaciones frecuentes inmediatas en paciente de 20-29 años sometidos a manga gástrica laparoscópica del Hospital Teodoro Maldonado}

Vol. 3, núm. 3., (2019)

Ana María Viteri Rojas; Katherine Angélica Sánchez Alcantara; Andrea Alexandra Saltos

Román

Marcuschamer, S. K., Castillo, J. R., Sanders, J. H., Murillo Zolezzi, A., Velasco, J. S., Iturbide, R. E., \& Etchegaray, A. (2009). Manga gástrica, manejo moderno del sobrepeso y la obesidad. Revista Mexicana de Cirugía Endoscópica, 10(1), 23-26.

Morales-Maza, J. (2018). Manga gástrica: Evidencia científica detrás de la técnica. Rev Mex de Cirugía del Aparato Digestivo,7(4), 128-133.

Moreno, G. M. (2012). Definición y clasificación de la obesidad. Revista Médica Clínica Las Condes, 23(2), 124-128.

Nassar, R. (2011). Gastrectomía vertical por laparoscopia "Manga gástrica". Revista Colombiana de Gastroenterología,26(4), 265-268.

Nutrición, C. N. (2011). Guías de práctica clínica para la prevención, el diagnóstico y el tratamiento de la obesidad. Arch argent pediatr, 109(3), 256-266.

Ochoa, D. C., Ortiz, V. P., Cedeño, J. P., Zambrano, R. G., Ortiz, F. J., \& Alonzo, G. C. (2018). Factores de Riesgo más relevantes en el aumento de obesidad infantil. RECIAMUC, 2(4), 24-40.

Ocón Bretón, J., Pérez Naranjo, S., Gimeno Laborda, S., Benito Ruesca, P., \& García Hernández, R. (2005). Eficacia y complicaciones de la cirugía bariátrica en el tratamiento de la obesidad mórbida. Nutrición Hospitalaria, 20(6), 409-414.

PARRA VILLEGAS, M. C. (2015). Cirugia de Manga Gastrica Complicaciones y Desventajas en el Hospital Doctor Teodoro Maldonado Carbo de junio a diciembre del 2013. Guayaquil, Ecuador\}: Trabajo de Grado - Universidad de Guayaquil, Facultad de Ciencias Médicas, Escuela de Medicina.

Rodríguez, M. F., Mora, P. G., \& Palacios, C. V. (2013). Obesidad mórbida y criterios de evaluación previos a la cirugía bariátrica. Trastornos de la conducta alimentaria, (18), 2008-2038.

Rodríguez-Esquerra, A., \& Beltrán-Gastélum, C. (2013). Comparación gastrectomía en manga laparoscópica Vs. bypass gástrico en Y de Roux en pérdida de peso. Arch Salud Sin, 7, 79-85.

San Lucas, Y. E., Montanero, E. E., Panchana, R. L., Robles, K. C., \& Vayas, D. A. (2018). Trastornos y enfermedades asociadas al consumo de azúcares. RECIAMUC,2(3), 295308.

Scarsella, C., \& Després, J. P. (2003). Tratamiento de la obesidad: necesidad de centrar la atención en los pacientes de alto riesgo caracterizados por la obesidad abdominal. Cadernos de Saúde Pública, 19, 7-19. 


\section{Complicaciones frecuentes inmediatas en paciente de 20-29 años sometidos a manga gástrica laparoscópica del Hospital Teodoro Maldonado}

Vol. 3, núm. 3., (2019)

Ana María Viteri Rojas; Katherine Angélica Sánchez Alcantara; Andrea Alexandra Saltos

Román

Solórzano, L. B., Flores, K. L., \& Ávila, L. E. (2019). Cirugía bariátrica como alternativa para la obesidad. RECIMUNDO, 3(2), 484-501.

Us de Paz, G., \& Contreras Parraguez, J. E. (2015). Complicaciones tardías en cirugía bariátrica. Rev. guatemalteca cir, 21(1), 85-100.

Velásquez, V. V., \& Alvarenga, J. C. (2001). Psicología y la obesidad. Revista de endocrinología y nutrición, 9(2), 91-96.

Viteri, A. M., Paredes, C. S., Pozo, S. D., Velásquez, G. M., López, A. B., \& Bravo, C. M. (2019). Avances en el tratamiento de la obesidad mórbida. RECIMUNDO, 3(2), 217-230.

Yeste, D., García, N., Gussinyer, S., Marhuenda, C., Clemente, M., Albisu, M., \& ... Carrascosa, A. (2008). Perspectivas actuales del tratamiento de la obesidad infantil. Revista Española de Obesidad, 6(3), 139-152. 